\title{
Nanomedicina y biomimetismo
}

\author{
Abraham Faustino-Vega* y Carlos Tomás Quirino-Barreda*
}

RESUMEN: Los sistemas materiales bioinspirados surgen a partir del mayor conocimiento de diferentes organismos vivos tales como: plantas, insectos, mamíferos y organismos marinos, por mencionar algunos. Estas formas de diseñar, procesar y ensamblar estructuras complejas para generar materiales altamente desarrollados con recursos mínimos pueden servir como modelo para diseñar sistemas de liberación de agentes terapéuticos (SLAT) con funciones óptimas. Los materiales bioinspirados que involucran el uso de superficies superhidrofóbicas y el ensamblado capa por capa están siendo implementados exitosamente para crear estructuras poliméricas o híbridas. Mimetizando la superficie, la forma, textura y movimientos de las células, dichos materiales pueden ayudar a superar el fenómeno de la fagocitosis y de esta forma hacer que los nanoacarreadores (vectores) permanezcan mayor tiempo dentro de la circulación sistémica para incrementar el control espaciotemporal de la liberación de agentes terapéuticos.

PALABRAS CLAVE: nanomedicina, biomimetismo, materiales bioinspirados, nanoacarreadores, vectorización

ABSTRACT: Bioinspired systems materials derived from different living organisms such as plants, insects, mammals and marine organisms, to mention a few. These forms of design, processing and assembling complex structures to generate materials that are highly developed with minimal resources can serve as a model for design drug delivery systems (DDS) with optimal features. Bioinspired materials that involve the use of superhydrophobic surfaces, layer by layer assembly are being successfully implemented in order to create a wide range of polymeric and hybrid structures. Mimicking the surface, shape, texture and movements of cells bioinspired materials can help to overcome the phenomenon of the phagocytosis and in this way make the nanocarriers remain more time within circulatory system to increase the control space-time of therapeutic agent's release.

KEYWORDS: nanomedicine, biomimetics, bio-inspired materials, nanocarriers, vectorization

\section{Introducción}

El término nanotecnología es utilizado para describir cualquier tecnología realizada a escala "nanométrica", donde un nanómetro equivale a una milmillonésima parte del metro (Ram et al., 2011).

La nanotecnología al aplicarse a la medicina se le conoce como nanomedicina, y constituye una gran oportunidad para llevar a cabo nuevos tratamientos o mejorar los ya existentes. Las diferentes herramientas de las que se vale la nanotecnología para manipular materiales a escala molecular y

* Laboratorio: Farmacia Molecular y Liberación Controlada, Unidad Interdisciplinaria de Docencia, Investigación y Servicio (UIDIS). Edificio “N”, Universidad Autónoma Metropolitana, Unidad Xochimilco. Calzada del Hueso 1100, Col. Villa Quietud, Delegación Coyoacán, 04960, México, D.F.

Correspondencia: (abraham.faustino@gmail.com) y (cquirino@correo.xoc.uam.mx). 
FIGURA 1. Ejemplos de nanopartículas con aplicación potencial en farmacia.

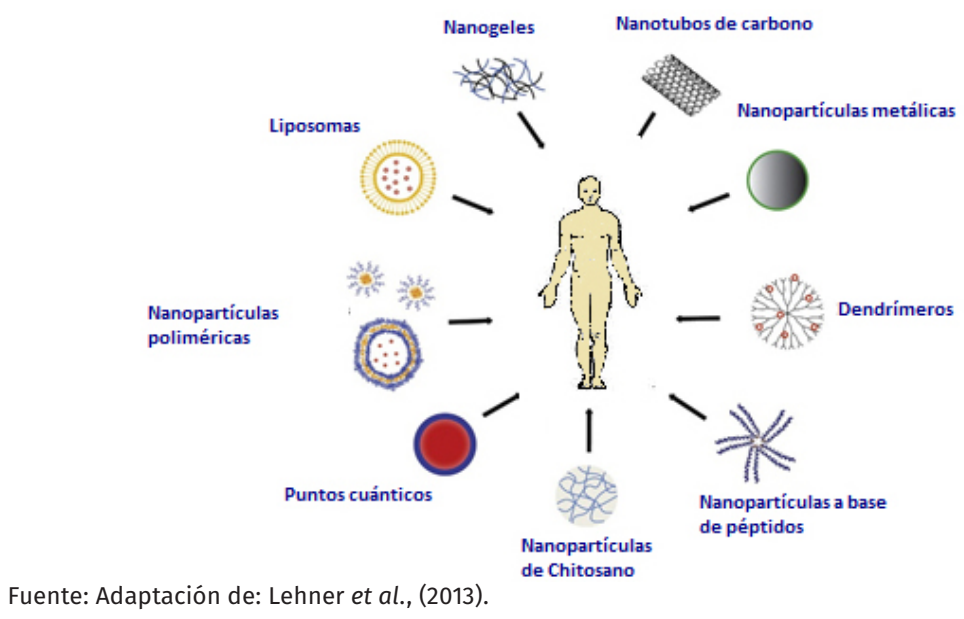

atómica para generar nanoestructuras artificiales que son del mismo tamaño que las biomoléculas y que pueden interaccionar con las células de los diferentes organismos vivos ofrecen nuevas soluciones en el diagnóstico, los tratamientos "inteligentes" y la medicina regenerativa; actuando y estimulando los propios mecanismos controladores y reparadores del cuerpo humano cuando se consigue que éstos actúen como nanoacarreadores o vectores nanométricos (Bhowmik et al., 2010, Moghimi et al., 2005, Kobayashi, 2007, Pierstorff y Ho, 2007).

Los vectores nanométricos son sistemas coloidales de talla submicrónica $(<1 \mathrm{~mm})$ que pueden ser fabricados a partir de una gran variedad de materiales y composiciones, los cuales incluyen, entre otros, liposomas, micelas, dendrímeros, nanopartículas sólidas lipídicas, nanopartículas metálicas, nanopartículas semiconductoras y nanopartículas poliméricas, puntos cuánticos, nanotubos, etc. (figura 1).

Los acarreadores nanométricos han sido considerados de gran interés en las últimas décadas como sistemas de liberación efectivos de fármacos por muchas razones, éstas incluyen (Ram et al., 2011):

i. Estabilidad física y biológica que puede facilitar el atrapamiento o la liberación controlada del fármaco.

ii. Buena tolerancia de los componentes.

iiii. Simplicidad del proceso de formulación y obtención.

iv. Posibilidad de escalar el proceso de fabricación.

Hoy día, gran parte de las nanopartículas desarrolladas para contener agentes terapéuticos (fármacos y biofármacos), encapsulados o adsorbidos, han mostrado su capacidad de dirigir la administración, minimizar los 
efectos colaterales y proteger a la sustancia activa durante su almacenamiento, liberación y transporte en el organismo. Al liberar el agente terapéutico solamente en el sitio origen de la enfermedad, se puede administrar bajas dosis de éste, ya que sólo una pequeña fracción de la sustancia activa puede perderse en otras partes del cuerpo. Así, fármacos tóxicos como los citostáticos sólo afectarán el tejido blanco y por lo tanto se pueden minimizar los efectos colaterales (Bhowmik et al., 2010; Marcato y Durán, 2008; Caldorera-Moore et al., 2010; Amin et al., 2011; Assadi et al., 2011; FernandezFernandez et al., 2011), tabla 1.

No obstante lo anterior, existen muchos obstáculos que las nanopartículas deben superar antes de conseguir la liberación del fármaco en el sitio blanco. Además, las nanopartículas deben ser eliminadas de forma segura del cuerpo, por ejemplo, mediante degradación o filtración a través del sistema renal. Otras características que deben reunir son la biocompatibilidad, la capacidad de liberar el fármaco de manera controlada y en el lugar deseado, así como evitar el reconocimiento y ataque por parte del sistema inmune. Algunas de estas propiedades pueden ser obtenidas al modificar su superficie y ciertos polímeros contenidos en la superficie de las nanopartículas pueden otorgarles la protección contra el sistema inmune. De igual forma, la capacidad de alcanzar un blanco predefinido puede lograrse al inmovilizar proteínas específicas en la superficie de las nanopartículas (Neal et al., 2009; Caldorera-Moore et al., 2010; Assadi et al., 2011; Tanner et al., 2011).

La funcionalización de la superficie de las nanopartículas ha sido extensamente explorada y ha probado ser un factor crítico en el desarrollo de nanopartículas para distintas aplicaciones prácticas. De manera particular, la funcionalización de la superficie de las nanopartículas ha sido extensamente

TABLA 1. Ventajas e inconvenientes de los sistemas de liberación modificada.

\begin{tabular}{|l|l|}
\hline VENTAJAS & DESVENTAJAS \\
\hline Baja dosis. & $\begin{array}{l}\text { Puede romperse la matriz y liberar todo } \\
\text { el contenido. }\end{array}$ \\
\hline Frecuencia baja de dosificación. & $\begin{array}{l}\text { Baja posibilidad para ajustar la dosis } \\
\text { una vez que ésta se ha establecido. }\end{array}$ \\
\hline $\begin{array}{l}\text { Mejor aceptación y cumplimiento por parte } \\
\text { del paciente. }\end{array}$ & $\begin{array}{l}\text { Es necesario que el paciente tenga } \\
\text { mayor instrucción operativa. }\end{array}$ \\
\hline $\begin{array}{l}\text { Menor fluctuación de los niveles plasmáticos } \\
\text { del fármaco. }\end{array}$ & $\begin{array}{l}\text { Generalmente estos sistemas son } \\
\text { costosos. }\end{array}$ \\
\hline $\begin{array}{l}\text { Mayor uniformidad en el efecto } \\
\text { farmacológico. }\end{array}$ & $\begin{array}{l}\text { Requieren de mayor especialización } \\
\text { para su desarrollo. }\end{array}$ \\
\hline Mayor relación de eficacia/seguridad. & \\
\hline
\end{tabular}


explorada para aplicaciones biológicas y biomédicas, tal es el caso de las denominadas sondas biomoleculares útiles en imagenología diagnóstica y en la liberación de agentes terapéuticos. En general, la funcionalización superficial con ligandos, polímeros y biomoléculas para impartir biofunciones específicas e incrementar la biocompatibilidad de las nanopartículas ha llegado a ser un componente esencial, y para estos sistemas nanoparticulados, la elección de uno o más agentes modificadores de la superficie también depende del tipo de efecto que se espera impartir, ya sea simple o múltiple. Por ejemplo, para evitar la aglomeración de las nanopartículas, pueden incorporarse en su superficie ligandos que contengan moléculas hidrofóbicas, mientras que las nanopartículas dirigidas para un uso en ambientes acuosos pueden requerir superficies recubiertas con polímeros hidrosolubles, tales como el polietilenglicol (PEG), que incrementará su solubilidad y biocompatibilidad. Por otra parte, los ligandos también pueden ser incorporados para ser utilizados como agentes de reconocimiento molecular superficial y de esta forma dirigir al activo terapéutico a su sitio blanco. Las estrategias comunes para la funcionalización superficial de las nanopartículas involucran la fisisorción no covalente y la bioafinidad o conjugación covalente con ligandos apropiados. (Nahar et al., 2006; Cigada, 2008; Marcato y Durán, 2008; Caldorera-Moore et al., 2010; Federici et al., 2012; Gao et al., 2013; Mahdavi et al., 2013).

Para la funcionalización de las superficies de las nanopartículas, las superficies de las membranas celulares han llegado a ser un gran modelo debido a sus propiedades intrínsecas de antiadhesión y a la actividad biológica específica presente a través de la superficie membranal. Por lo tanto, la construcción de una estructura membranal celular artificial basada en fosfolípidos se ha convertido en una herramienta efectiva en la prevención de las reacciones biológicas no específicas a que pueden dar lugar las superficies de las nanopartículas en desarrollo. Además, ciertas moléculas bioactivas pueden ser inmovilizadas en la superficie de los fosfolípidos para generar nanopartículas biológicamente activas más representativas de las superficies membranales celulares (Mahdavi et al., 2013).

\section{Sistemas de liberación de agentes terapéuticos}

Las formas farmacéuticas novedosas también denominadas sistemas de liberación de agentes terapéuticos (SLAT) son concebidas ahora no sólo para comportarse como facilitadores del almacenamiento, transporte, manipulación, administración y regulación de la liberación local o sistémica del fármaco, sino también para controlar el microambiente a través del cual el fármaco tiene que transitar y difundir en su ruta hacia el sitio de acción, de modo que el fármaco obtendrá con ello la ayuda necesaria para superar ciertas barreras y establecer el proceso de liberación a ciertas demandas específicas. De la misma manera, los SLAT también pueden ser concebidos como verdaderos 
sistemas acarreadores o de transporte, con la capacidad adicional de liberar el fármaco en las mejores condiciones posibles de absorción y con influencia en la modulación de la distribución y aclaramiento de los principios activos en el cuerpo. Un SLAT avanzado deberá comportarse como un medio de "autoliberación", es decir, permitir la liberación dirigida con niveles terapéuticos deseados a los órganos, tejidos y estructuras celulares específicas, cuando esto sea necesario (Yoo et al., 2011; Singh et al., 2012; Cigada, 2008; Huang y Juluri, 2008; Xu et al., 2008; Carmona-Ribeiro, 2010; Xu et al., 2010; Zhang et al., 2010; Al-kattan et al., 2011).

Los procesos de transporte y reconocimiento dentro del cuerpo humano y animal sirven como modelos excelentes para el diseño de los SLAT. La evolución de la tecnología farmacéutica hacia la nanotecnología ha llevado a generar sistemas más eficientes con sólo combinar algunas biomoléculas como aminoácidos, azúcares y lípidos. Las biomoléculas en los organismos vivos desarrollan funciones increíblemente complejas, adoptando arquitecturas que son el resultado del ensamblado correcto de los componentes que interactúan con alta especificidad a escalas extremadamente pequeñas. Es por lo anterior que, si bien hoy en día la química y la biotecnología ofrecen millones de compuestos que pueden ser usados como componentes de otros compuestos de síntesis o de conformación estructural no necesariamente a base de uniones covalentes o iónicas, encontrar la secuencia y conformación apropiada es la clave para generar los denominados sistemas biomiméticos.

El término biomimética se utiliza para describir la imitación de los sistemas y diseños biológicos. Es un campo emergente de la ciencia que incluye el estudio de cómo la naturaleza, diseña, procesa, ensambla y desensambla bloques moleculares para fabricar materiales suaves altamente desarrollados y compósitos minerales, para entonces aplicar estos diseños y procesos en la construcción de moléculas nuevas y materiales con propiedades únicas. Mimetizar moléculas selectivas, camuflar los recubrimientos o componentes estímulosensibles son estrategias en la actualidad con un alto impacto en el diseño de los SLAT e involucran un trabajo interdisciplinario (Ranney, 2000; Prokop, 2001; Xu et al., 2008; Bhushan, 2009; Carmona-Ribeiro, 2010; Haidar, 2010; Yoo et al., 2011; Singh et al., 2012). La aplicación de los principios biomiméticos no sólo puede generar sistemas acarreadores de fármaco con estructuras y funcionalidad bioinspiradas, sino también nuevos caminos para fabricar distintos sistemas acarreadores.

\section{Procedimientos bioinspirados para elaborar SLAT}

Los métodos convencionales de encapsulación (evaporación, extracción, coacervación, polimerización interfacial, entre otros), en general involucran dos o más fases y condiciones un poco drásticas para lograr la encapsulación de la o las moléculas de interés, los cuales pueden comprometer la estabilidad de fármacos lábiles y también complicar el rendimiento de la 
encapsulación debido a la migración/difusión de la fase líquida externa. Sin embargo, el análisis más detallado de las superficies superhidrofóbicas de ciertas hojas de plantas y alas de insectos ha dado lugar en la actualidad a la implementación de técnicas en procesos de tratamiento superficial que reproduce sus características en materiales sintéticos y con ello se consiguen mejores recubrimientos (Carmona-Ribeiro, 2010; Yoo et al., 2011; Singh et al., 2012).

Las células epidérmicas papilosas de la hoja de la flor de loto forman rugosidades a escala microscópica y nanoscópica. La superficie de estas hojas está cubierta con diversas ceras integradas por una mezcla de compuestos hidrocarbonados que la dotan de una fuerte repulsión al mojado, haciendo que las pequeñas gotas de agua que caen en su superficie, se asienten en la punta de las nanoestructuras, mientras las burbujas de aire se disponen en los valles. De tal forma, así como las gotas de agua resbalan sobre las hojas de loto, la dispersión de monómeros/polímeros que contiene el fármaco puede ser aplicada (con una pipeta o una aguja) en superficies superhidrofóbicas de cobre, aluminio o poliestireno, y con ello generar, después de la polimerización o entrecruzamiento, partículas prácticamente esféricas, y por tener lugar el proceso en la interface sólido/gas no hay migración del fármaco; entonces, es posible alcanzar un alto rendimiento de encapsulación. La versatilidad de esta aproximación ha sido demostrada por preparación de microgeles de poli-( $\mathrm{N}$-isopropilacrilamida) y dextrano que encapsula proteínas y puede controlar la liberación por varias horas. Al depositar gotas adicionales en los micro o nanogeles preformados lleva a sistemas multicapas para la modulación simultánea de liberación de varias sustancias terapéuticas (Prokop, 2001; Pierstorff y Ho, 2007; Cigada, 2008; Huang y Juluri, 2008; Mrsny, 2009).

Por otro lado, la técnica de aproximación capa por capa $(\mathrm{CPC})$ mimetiza típicamente la manera en que la naturaleza sigue un proceso para construir una variedad de estructuras de abajo hacia arriba. A través de combinaciones versátiles de interacción de fuerzas (electrostáticas, hidrofóbicas, puentes de hidrógeno o de reconocimiento biológico) y de materiales (polímeros, péptidos y nanopartículas), es posible controlar finamente la localización y concentración de los componentes en los acarreadores de fármacos a escala nanométrica. La liberación de las sustancias terapéuticas atrapadas en las capas depende de la permeabilidad o el rompimiento de las estructuras multicapas, y pueden ser programadas para desintegrar o disparar la disolución del ensamblado cuando se aplica un estímulo.

Las pruebas in vivo han demostrado el enorme potencial de los CPC al utilizar fármacos, genes y vacunas, incrementando notablemente la biodisponibilidad de los compuestos quimioterapéuticos. Por otra parte, el mimetizar la morfogénesis natural de tejidos duros tales como conchas de moluscos o dientes o huesos humanos, puede generar materiales híbridos. Las proteínas que controlan la deposición de fosfatos o sílice en los procesos 
naturales, también pueden ayudar a regular la composición, geometría y estructura de macro y microporos de compósitos inorgánicos disponibles para liberar materiales prostéticos y nanovehículos sistémicos (Yoo et al., 2011; Singh et al., 2012).

\section{Superficies bioinspiradas para los nanoacarreadores}

El éxito clínico de los nanoacarreadores está limitado por los eventos adversos que ocurren en el ambiente biológico: en la sangre puede haber fenómenos como la opsonización y precipitación por las proteínas, que pueden hacer no alcanzar a los nanoacarreadores el endotelio; por otra parte, son tejidos ricos en lípidos y además presentan alguna patología, pueden comprometer la estabilidad de los nanoacarreadores. Para superar los receptores de la superficie endotelial de tejidos dañados y conferirle afinidad hacia los anticuerpos, pueden utilizarse componentes de leucocitos, bacterias y virus, y de esta forma alcanzar el endotelio y más adelante llegar al órgano blanco. El sulfato de glucosamina puede actuar como un polímero biomimético que puede adherirse a las adhesinas vasculares como lo hacen los leucocitos. Las nanopartículas recubiertas con este material son repelidas por el endotelio normal, pero son selectivamente adheridas por el endotelio de células tumorales y de esta forma cruzarlo para llegar al tejido tumoral.

Asimismo, son particularmente atractivos los ligandos que mimetizan la forma utilizada por los microrganismos para reconocer e internalizarse en las células. Los adenovirus citolíticos y proenzimas de la salmonella spp penetran y replican preferentemente dentro de células tumorales, destruyendo el tejido y deteniéndose en los límites del tumor. Un virus oncolítico modificado genéticamente, combinado con fármacos quimioterapéuticos, fue aprobado en 2005 para tratar cáncer de cabeza y cuello. Sin embargo, las interacciones complejas entre el tumor, el virus y el sistema inmune son aún obstáculos para la terapia oncolítica (Haidar, 2010; Al-kattan et al., 2011; Kramer, 2011; Yoo et al., 2011; Brunner et al., 2012; Lapidot et al., 2012; Singh et al., 2012).

\section{Forma y textura bioinspiradas}

La mayoría de los nanoacarreadores tienen formas esféricas y rígidas. Para mejorar el desempeño durante la fagocitosis, la forma de éstos puede hacerse más suave y cilíndrica. Los SLAT basados en eritrocitos y células dentríticas ofrecen buenas perspectivas. Los eritrocitos con cubierta antifagocitosis CD47 han mostrado ser útiles para transportar fármacos dispersados en su interior o conjugados en su superficie. Por otro lado, los SLAT mucoadhesivos, basados en componentes bacteriales flagelares, pueden usarse para adherirse a las mucosas humanas. Otro modelo es mimetizar las almohadillas adhesivas del geco para generar nanoacarreadores que puedan adherirse a 
las células aún en presencia de grandes volúmenes de líquido (Ranney, 2000; Prokop, 2001; Lapidot et al., 2012; Gao et al., 2013; Weingart et al., 2013).

\section{Estructuras internas bioinspiradas}

Todas las estrategias antes mencionadas tratan de mimetizar los mecanismos fisiológicos de reconocimiento y retroalimentación, y su diseño está basado en los procesos de autoensamblado y construcción de acarreadores biológicos. En el caso de los SLAT, una vez que el nanoacarreador llega al sitio blanco, debe liberar adecuadamente el agente terapéutico. Los componentes que reconocen inequívocamente ciertas variables fisicoquímicas del microambiente ( $\mathrm{pH}$, temperatura o concentración) disparan el mecanismo de liberación diseñado para tal fin. La velocidad de liberación del agente terapéutico puede ser regulada externamente con ultrasonido, luz o campo magnético. Las proteínas son hasta ahora los modelos más apropiados para tal fin, pues poseen ventajas únicas como: reconocimiento molecular, autoensamblado y disponibilidad para la manipulación genética.

Los avances en la biotecnología y la genómica llevan a la incorporación de secuencia de péptidos responsables de funciones específicas dentro de los nanoacarreadores. Un material puede ser diseñado mimetizando regiones hidrofóbicas o hidrofílicas de un péptido, de esta forma la región hidrofóbica de la molécula se ensamblará con otras moléculas similares, en tanto que la región hidrofílica será responsable de adecuarse con el ambiente biológico, ejemplos de éstos pueden ser las micelas, vesículas y túbulos. Varias biomacromoléculas como anticuerpos, leucina, colágeno o cápsides virales desarrollan su función cuando se ensamblan, pero están inactivas cuando se disocian. Similar a lo anterior, las estructuras ensambladas por algún estímulo pueden actuar como nanoacarreadores dentro del flujo sanguíneo cuando se asocian con micelas.

Así como la estructura de los receptores farmacológicos es utilizada para descubrir o diseñar nuevos fármacos, este procedimiento también puede ser aplicado como una guía para diseñar dominios activos utilizando monómeros que contengan ciertos grupos funcionales similares a los aminoácidos que están presentes en el sitio activo del receptor. A manera de ejemplo, esta aproximación ha sido explotada para desarrollar lentes de contacto para liberar agentes antihistamínicos e inhibidores de la anhidrasa carbónica en la superficie ocular (Ranney, 2000; Prokop, 2001; Lapidot et al., 2012; Gao et al., 2013; Weingart et al., 2013).

\section{Movimientos biomiméticos}

El paso de los nanoacarreadores a través del cuerpo es tan difícil como atravesar una calle transitada. Mimetizar el movimiento de los organismos vivos y de ciertas células puede servir como modelo para predecir la capacidad de 
TABLA 2. Ejemplos de sistemas bioinspirados con aplicación potencial en medicina.

\begin{tabular}{|c|c|c|c|}
\hline SISTEMA & MATERIAL UTILIZADO & APLICACIÓN & REFERENCIA \\
\hline $\begin{array}{l}\text { Nanopartícula } \\
\text { magnética }\end{array}$ & $\begin{array}{l}\text { DSPE, DOPE, DMPE, DPPE, } \\
\text { DSPE-PEGX }\end{array}$ & $\begin{array}{l}\text { Agente de contraste MRI } \\
\text { Liberación de fármaco/gen }\end{array}$ & $\begin{array}{l}\text { (Nitin et al., 2004) } \\
\text { (Lee et al., 2012) } \\
\text { (Glaus et al., 2010) }\end{array}$ \\
\hline $\begin{array}{l}\text { Nanopartícula } \\
\text { polimérica }\end{array}$ & $\begin{array}{l}\text { DSPE, DOPE, DMPE } \\
\text { DSPE-PEGX }\end{array}$ & Liberación de fármacos & $\begin{array}{l}\text { (Huo et al., 2006) } \\
\text { (Ahmed y Wunder, 2009) }\end{array}$ \\
\hline $\begin{array}{l}\text { Nanopartícula } \\
\text { polimérica }\end{array}$ & $\begin{array}{l}\text { Glicosaminoglicano } \\
\text { aniónico y DS } 435\end{array}$ & $\begin{array}{l}\text { Liberación de fármacos } \\
\text { antitumoral y como agente } \\
\text { de contraste }\end{array}$ & $\begin{array}{l}\text { (Ranney et al., 2005) } \\
\text { (Said Hassane et al., 2010) }\end{array}$ \\
\hline $\begin{array}{l}\text { Nanopartícula } \\
\text { polimérica }\end{array}$ & Membrana de eritrocito & Liberación de fármacos & $\begin{array}{l}\text { (Hu et al., 2011) } \\
\text { (Merkel et al., 2011) }\end{array}$ \\
\hline $\begin{array}{l}\text { Nanopartícula } \\
\text { magnética }\end{array}$ & $\begin{array}{l}\text { Poli[n-isopropilacrilamida] } \\
\text { Poli-NIPAm }\end{array}$ & $\begin{array}{l}\text { Liberación de fármacos } \\
\text { antitumoral y como agente } \\
\text { de contraste }\end{array}$ & $\begin{array}{l}\text { (Timko y Kohane, 2012) } \\
\text { (Altunbas y Pochan, 2012) }\end{array}$ \\
\hline $\begin{array}{l}\text { Nanopartículas } \\
\text { inorgánicas y } \\
\text { nanofibras }\end{array}$ & $\begin{array}{l}\text { Dióxido de silicio } \\
\text { Celulosa }\end{array}$ & $\begin{array}{l}\text { Aplicación en microfluidos } \\
\text { y liberación de fármacos }\end{array}$ & $\begin{array}{l}\text { (Ghost y Fischer, 2009) } \\
\text { (Jin et al., 2011) }\end{array}$ \\
\hline
\end{tabular}

alcanzar los tejidos. Los hidrogeles hechos de polietilenglicol y de polímeros iónicos electroatractivos o de dióxido de silicio, en combinación con señales eléctricas y magnéticas, pueden ser transformados en minirrobots capaces de simular el movimiento de los pulpos, miriápodos, espermatozoides o flagelos bacteriales. Los avances recientes en aerogeles superhidrofóbicos y superhidrofílicos, pueden llevar a la fabricación de SLAT con facilidad de movimiento a través de las interfaces agua-aceite. También se han obtenido redes autopulsantes que imitan la oscilación autónoma del corazón, ondas cerebrales o secreción pulsátil de hormonas, usando la conversión de ADP en ATP o el ciclo de Krebs. Estos hidrogeles autoscilantes son interesantes como SLAT capaces de liberar agentes terapéuticos cíclicamente imitando los biorritmos (Cigada, 2008; Huang y Juluri, 2008; Xu et al., 2008; Carmona-Ribeiro, 2010; Haidar, 2010; Xu et al., 2010; Zhang et al., 2010; Al-kattan et al., 2011; Kramer, 2011; Yoo et al., 2011; Brunner et al., 2012; Gong y Winnik, 2012; Lapidot et al., 2012; Singh et al., 2012; Weingart et al., 2013).

\section{Conclusiones}

Aprovechar la ingeniería de la naturaleza de millones de años de experiencia para innovar, es lo que conocemos como biomimética. La naturaleza ya ha descubierto qué hacer para sobrevivir, pues nos da lecciones de comportamiento. Por eso, por el hecho de que aún no sabemos mucho de ella, debemos observarla, entenderla e imitarla y aplicar estos conocimientos en el campo de la nanomedicina. Mimetizar la manera en cómo las sustancias y 
las células son enviadas de un lugar a otro dentro de los organismos vivos, así como comprender la manera en que interactúan con otros elementos fisiológicos o en que disparan respuestas específicas, puede incrementar notablemente la eficacia y seguridad de los nuevos tratamientos médicos apoyados con los SLAT. La modificación superficial de los nanoacarreadores puede hacer que éstos tengan éxito al cruzar las diferentes barreras biológicas y seleccionar los tejidos blancos dañados. Los nanoacarreadores inspirados en las células han mostrado una mejor resistencia contra la fagocitosis. Además, los componentes de estímulo-respuesta tales como los recubrimientos tipo membrana ofrecen mecanismos novedosos para controlar la liberación de los agentes terapéuticos en el sitio de acción. De tal forma, aunque todavía escasos, algunos sistemas bioinspirados ya han demostrado su potencial en el área clínica. Así, la biomimética ofrece una gran oportunidad para mejorar o generar los sistemas novedosos de liberación de agentes terapéuticos que promete la nanomedicina, lo cual es el resultado de integrar el conocimiento aportado por las diversas disciplinas que estudian todo el cúmulo de enseñanzas que la naturaleza nos brinda.

\section{Bibliografía}

Ahmed, S. y Wunder, S. L. (2009) Effect of high surface curvature on the main phase transition of supported phospholipid bilayers on $\mathrm{SiO} 2$ nanoparticles. Langmuir, 25: 3682-3691.

Al-Kattan, A., Dufour, P. y Drouet, C. (2011) Purification of biomimetic apatitebased hybrid colloids intended for biomedical applications: A dialysis study. Colloids and Surfaces B: Biointerfaces, 82: 378-384.

Altunbas, A. y Pochan, D. J. (2012) Peptide-based and polypeptide-based hydrogels for drug delivery and tissue engineering. Pub Med, 310: 135-167.

Amin, R., Hwang, S. y Park, S. H. (2011) Nanobiotechnology: An interface between nanotechnology and biotechnology. Nano, 6: 101-111.

Assadi, M., Afrasiabi, K., Nabipour, I. y Seyedabadi, M. (2011) Nanotechnology and nuclear medicine; research and preclinical applications. Hellenic Journal of Nuclear Medicine, 14: 149-159+197-198.

Bhowmik, D., Chiranjib, Margret Chandira, R., Tripathi, K. K. y Sampath Kumar, K. P. (2010) Nanomedicine-an overview. International Journal of PharmTech Research, 2: 2143-2151.

Bhushan, B. (2009) Biomimetics: Lessons from Nature - an overview. Philosophical Transactions of the Royal Society A: Mathematical, Physical and Engineering Sciences, 367: 1445-1486.

Brunner, R., Sandfuchs, O., Pacholski, C., Morhard, C. y Spatz, J. (2012) Lessons from nature: Biomimetic subwavelength structures for high-performance optics. Laser and Photonics Reviews, 6: 641-659.

Caldorera-Moore, M., Guimard, N., Shi, L. y Roy, K. (2010) Designer nanoparticles: Incorporating size, shape and triggered release into nanoscale drug carriers. 
Expert Opinion on Drug Delivery, 7: 479-495.

Carmona-Ribeiro, A. M. (2010) Biomimetic nanoparticles: Preparation, characterization and biomedical applications. International Journal of Nanomedicine, 5: 249-259.

Cigada, A. (2008) Biomaterials, tissue engineering, gene therapy. Journal of Applied Biomaterials and Biomechanics, 6: 127-131.

Federici, S., Oliviero, G., Maiolo, D., Depero, L. E., Colombo, I. y Bergese, P. (2012) On the thermodynamics of biomolecule surface transformations. Journal of Colloid and Interface Science, 375: 1-11.

Fernandez-Fernandez, A., Manchanda, R. y McGoron, A. J. (2011) Theranostic applications of nanomaterials in cancer: Drug delivery, image-guided therapy, and multifunctional platforms. Applied Biochemistry and Biotechnology, 165: 1628-1651.

Gao, W., Hu, C. M. J., Fang, R. H., Luk, B. T., Su, J. y Zhang, L. (2013) Surface functionalization of gold nanoparticles with red blood cell membranes. Advanced Materials, 25: 3549-3553.

Ghost, A. y Fischer, P. (2009) Controlled propulsion of artificial magnetic nanostructured propellers. Nano Letters, 9: 2243-2245.

Glaus, C., Rossin, R., Welch, M. J. y Bao, G. (2010) In vivo evaluation of 64Cu-labeled magnetic nanoparticles as a dual-modality PET/MR imaging agent. Bioconjugate Chemistry, 21: 715-722.

Gong, Y. K. y Winnik, F. M. (2012) Strategies in biomimetic surface engineering of nanoparticles for biomedical applications. Nanoscale, 4: 360-368.

Haidar, Z. S. (2010) Bio-inspired/-functional colloidal core-shell polymeric-based nanosystems: Technology promise in tissue engineering, bioimaging and nanomedicine. Polymers, 2: 323-352.

Hu, C. M. J., Zhang, L., Aryal, S., Cheung, C., Fang, R. H. y Zhang, L. (2011) Erythrocyte membrane-camouflaged polymeric nanoparticles as a biomimetic delivery platform. Proceedings of the National Academy of Sciences of the United States of America, 108: 10980-10985.

Huang, T. J. y Juluri, B. K. (2008) Biological and biomimetic molecular machines. Nanomedicine, 3: 107-124.

Huo, Q., Liu, J., Wang, L. Q., Jiang, Y., Lambert, T. N. y Fang, E. (2006) A new class of silica cross-linked micellar core-shell nanoparticles. Journal of the American Chemical Society, 128: 6447-6453.

Jin, H., Kettunen, M., Laiho, A., Pynnönen, H., Paltakari, J., Marmur, A., Ikkala, O. y Ras, R. H. A.(2011) Superhydrophobic and superoleophobic nanocellulose aerogel membranes as bioinspired cargo carriers on water and oil. Langmuir, 27: 1930-1934.

Kobayashi, H. (2007) Molecular imaging; current status and future prospects in USA. Nippon rinsho. Japanese journal of clinical medicine, 65, 204-210.

Kramer, W. (2011). Transporters, Trojan horses and therapeutics: Suitability of bile acid and peptide transporters for drug delivery. Biological Chemistry, 392: 77-94. 
Lapidot, S., Meirovitch, S., Sharon, S., Heyman, A., Kaplan, D. L. y Shoseyov, O. (2012) Clues for biomimetics from natural composite materials. Nanomedicine, 7: 1409-1423.

Lee, N., Choi, Y., Lee, Y., Park, M., Moon, W. K., Choi, S. H. y Hyeon, T. (2012) Waterdispersible ferrimagnetic iron oxide nanocubes with extremely high $\mathrm{r} 2$ relaxivity for highly sensitive in vivo MRI of tumors. Nano Letters, 12: 3127-3131.

Lehner, R., Wang, X., Marsch, S. y Hunziker, P. (2013) Intelligent nanomaterials for medicine: Carrier platforms and targeting strategies in the context of clinical application. Nanomedicine: Nanotechnology, Biology, and Medicine, 9: 742-757.

Mahdavi, M., Ahmad, M. B., Haron, M. J., Namvar, F., Nadi, B., Ab Rahman, M. Z. y Amin, J. (2013) Synthesis, surface modification and characterisation of biocompatible magnetic iron oxide nanoparticles for biomedical applications. Molecules, 18: 7533-7548.

Marcato, P. D. y Durán, N. (2008) New aspects of nanopharmaceutical delivery systems. Journal of Nanoscience and Nanotechnology, 8: 2216-2229.

Merkel, T. J., Jones, S. W., Herlihy, K. P., Kersey, F. R., Shields, A. R., Napier, M., Luft, J. C., Wu, H., Zamboni, W. C., Wang, A. Z., Bear, J. E. y Desimone, J. M. (2011) Using mechanobiological mimicry of red blood cells to extend circulation times of hydrogel microparticles. Proceedings of the National Academy of Sciences of the United States of America, 108: 586-591.

Moghimi, S. M., Hunter, A. C. y Murray, J. C. (2005) Nanomedicine: Current status and future prospects. FASEB Journal, 19: 311-330.

Mrsny, R. J. (2009) Lessons from nature: "Pathogen-Mimetic" systems for mucosal Nano-medicines. Advanced Drug Delivery Reviews, 61: 172-192.

Nahar, M., Dutta, T., Murugesan, S., Asthana, A., Mishra, D., Rajkumar, V., Tare, M., Saraf, S. y Jain, N. K. (2006) Functional polymeric nanoparticles: An efficient and promising tool for active delivery of bioactives. Critical Reviews in Therapeutic Drug Carrier Systems, 23: 259-318.

Neal, R. A., Oredein-McCoy, O. y Botchwey, E. A. (2009) Nanomedicine: Addressing cardiovascular disease and cardiovascular tissue regeneration. Current Bioactive Compounds, 5: 206-214.

Nitin, N., Laconte, L. E. W., Zurkiya, O., Hu, X. y Bao, G. (2004) Functionalization and peptide-based delivery of magnetic nanoparticles as an intracellular MRI contrast agent. Journal of Biological Inorganic Chemistry, 9: 706-712.

Pierstorff, E. y Ho, D. (2007) Monitoring, diagnostic, and therapeutic technologies for nanoscale medicine. Journal of Nanoscience and Nanotechnology, 7: 29492968.

Prokop, A. (2001) Bioartificial organs in the twenty-first century: Nanobiological devices. Annals of the New York Academy of Sciences, 944: 472-490.

Ram, Y. M., Yadav, H. K. S., Singh, M. N. y Shivakumar, H. G. (2011) Nanoparticles, promising carriers in drug targeting: A review. Current Drug Therapy, 6: 87-96.

Ranney, D., Antich, P., Dadey, E., Mason, R., Kulkarni, P., Singh, O., Chen, H., Constantanescu, A. y Parkey, R. (2005) Dermatan carriers for neovascular transport targeting, deep tumor penetration and improved therapy. Journal of 
Controlled Release, 109: 222-235.

Ranney, D. F. (2000) Biomimetic transport and rational drug delivery. Biochemical Pharmacology, 59: 105-114.

Said Hassane, F., Saleh, A. F., Abes, R., Gait, M. J. y Lebleu, B. (2010) Cell penetrating peptides: Overview and applications to the delivery of oligonucleotides. Cellular and Molecular Life Sciences, 67: 715-726.

Singh, A. V., Rahman, A., Sudhir Kumar, N. V. G., Aditi, A. S., Galluzzi, M., Bovio, S., Barozzi, S., Montani, E. y Parazzoli, D. (2012) Bio-inspired approaches to design smart fabrics. Materials and Design, 36: 829-839.

Tanner, P., Baumann, P., Enea, R., Onaca, O., Palivan, C. y Meier, W. (2011) Polymeric vesicles: From drug carriers to nanoreactors and artificial organelles. Accounts of Chemical Research, 44: 1039-1049.

Timko, B. P. y Kohane, D. S. (2012) Materials to Clinical Devices: Technologies for Remotely Triggered Drug Delivery. Clinical Therapeutics, 34: S25-S35.

Weingart, J., Vabbilisetty, P. y Sun, X. L.(2013) Membrane mimetic surface functionalization of nanoparticles: Methods and applications. Advances in Colloid and Interface Science, 197-198: 68-84.

Xu, J., Sigworth, F. J. y Lavan, D. A. (2010) Synthetic protocells to mimic and test cell function. Advanced Materials, 22: 120-127.

Xu, J. P., Jin, Q., Zhu, L. Z., Ji, J. y Shen, J. C. (2008) Bioinspired phosphorylcholine motivation for biocompatible monolayer protected carbon nanotubes (CNTs) in nanomedicine. Journal of Bionanoscience, 2: 49-53.

Yoo, J. W., Irvine, D. J., Discher, D. E. y Mitragotri, S. (2011) Bio-inspired, bioengineered and biomimetic drug delivery carriers. Nature Reviews Drug Discovery, 10: 521-535.

Zhang, Z., Chen, J., Ding, L., Jin, H., Lovell, J. F., Corbin, I. R., Cao, W., Lo, P. C., Yang, M., Tsao, M. S., Luo, Q. y Zheng, G. (2010) HDL-mimicking peptide-lipid nanoparticles with improved tumor targeting. Small, 6: 430-437. 